\title{
SALA DE AULA INVERTIDA NA EDUCAÇÃO PARA AS PROFISSÕES DE SAÚDE: CONCEITOS ESSENCIAIS PARA A PRÁTICA
}

Flipped classroom in undergraduate health professions education: key concepts to the practice

Submetido em: 16/08/2016

La clase al revés en la educación para las profesiones de salud: conceptos clave para la práctica

Aceito em: 07/12/2016

Valdes Roberto Bollela1*, Mario Luís Ribeiro Cesaretti

Publicado em: 30/03/2017

'Faculdade de Medicina de Ribeirão Preto, Universidade de São Paulo

2Pontifícia Universidade Católica de São Paulo

*E-mail: vbollela@fmrp.usp.br

\section{Resumo}

As diretrizes curriculares dos cursos de graduação da área da saúde recomendam a utilização de estratégias de ensino e aprendizagem centradas no estudante, colaborativas e que valorizem o reconhecimento e a resolução de problemas relevantes e relacionados à prática profissional. O objetivo deste artigo é descrever os aspectos essenciais sobre a "sala de aula invertida", com o intuito de auxiliar aqueles que tiverem interesse de utilizá-la. Trata-se de breve revisão sobre uma estratégia educacional que tem sido empregada cada vez mais no ensino superior, em especial nos cursos de graduação da área da saúde. Os autores apresentam uma breve revisão sobre as origens, caracterizando e descrevendo como funciona a sala de aula invertida, além de discutir algumas razões para que o professor considere utilizá-la no ensino de graduação dos cursos da área da saúde. Finalmente, são apresentadas as limitações e os desafios da estratégia. A proposta da sala de aula invertida entrelaça diversos conceitos, dentre eles o da aprendizagem ativa e colaborativa, da autonomia do estudante, da aprendizagem significativa e do ensino à distância.

Palavras-chave: Educação em saúde, Educação à distância, Aprendizagem ativa.

\begin{abstract}
Brazilian curriculum guidelines for undergraduate health professions education recommend the use of student-centered, collaborative teaching and learning strategies that value the recognition and resolution of relevant problems related to professional practice. This article describes the essential aspects of flipped classroom in order to serve as a guide for those who want to try it. It is a brief review on a topic that has been gaining momentum and usage in graduate education, especially in the health professions where it might really fit for purpose. The authors present a brief review of the flipped classroom roots, showing how it works and the reasons that may lead a teacher to consider using it in health professions undergraduate courses. Finally, the limitations and challenges are presented. Flipped classroom connects several educational concepts, including the active, meaningful and collaborative learning, and fosters students' autonomy mixing in site and distance learning.
\end{abstract}

Keywords: Health education, Distance education, Active learning. 


\section{Resumen}

Las directrices curriculares de los cursos de grado del área de salud recomiendan el uso de estrategias de enseñanza y de aprendizaje centradas en el estudiante, colaborativas y que valoran el reconocimiento y la solución de problemas importantes relacionados a la práctica profesional. El objetivo de este artículo es describir los aspectos esenciales de "la clase al revés" con el fin de servir como guía para aquellos que quieran utilizarla. Es una breve revisión sobre esa estrategia educativa de enseñanza que ha ido ganando impulso cada vez más en la educación superior, especialmente, en los cursos de grado del área de salud. Los autores presentan una breve revisión sobre su origen, caracterizando y describiendo como funciona la clase al revés, además de discutir algunas razones importantes para que el profesor considere usarla en la enseñanza de los cursos de grado del área de salud. Por último, se presentan las limitaciones y desafíos de la estrategia. La propuesta de la clase al revés conecta varios conceptos educativos, incluyendo el aprendizaje activo y colaborativo, la autonomía del estudiante, el aprendizaje significativo y la enseñanza a distancia.

Palabras clave: Educación en salud, Educación a distancia, Aprendizaje activo.

\section{INTRODUÇÃO:}

Até recentemente, na educação das profissões da saúde, prevalecia o conceito de que "bastaria ser um excelente profissional que, consequentemente, o indivíduo seria também um excepcional professor". Felizmente, isto está mudando e crescem as oportunidades de desenvolvimento docente ofertadas pelas instituições de ensino superior, ao mesmo tempo em que aumenta o interesse do professor para investir no seu próprio desenvolvimento como educador ${ }^{(1)}$.

Essa postura aberta ao aprendizado, por parte do professor, é vital à sua atualização para a prática educacional, além de acrescentar novas estratégias de ensino/aprendizagem ao que podemos denominar de "caixa de ferramentas do educador". Quanto mais rico e diverso for o conteúdo da "caixa", mais recursos terá o educador para auxiliar seus estudantes a aprenderem e tornarem-se profissionais competentes.

Este artigo sobre a "sala de aula invertida" (do inglês flipped-classroom) tem como objetivo descrever os aspectos essenciais dessa estratégia, com o intuito de auxiliar professores que tenham interesse em utilizá-la.

A forma habitual de conduzir atividades educacionais no ensino superior é aquela em que o professor utiliza o tempo presencial da aula com os alunos para fazer apresentações sobre o tema do curso/disciplina, geralmente seguido de tarefas (exercícios e problemas) complementares para serem realizados pelo estudante. Este é o modelo em que o aprendizado está mais centrado no professor ${ }^{(2)}$.

Na área da saúde, é comum os estudantes terem aulas teóricas seguidas de atividades práticas, em que vivenciam aspectos relacionados ao que está sendo ensinado em sala de aula. Muitas vezes, os estudantes têm dificuldade para identificar a correlação entre a teoria e a sua relevância para a prática profissional. Como agravante, no momento do estudo individual, quando surgem as dúvidas, o professor não está presente para esclarecê-las.

Por exemplo, nos cursos de Medicina que utilizam a aula teórica como a principal estratégia de ensino, a regra é o estudante ter aulas com o professor, realizar práticas em laboratórios ou em cenários de prática e, ao final da disciplina, ser avaliado em provas parciais e/ou finais. Normalmente, existem poucas oportunidades para que os estudantes apliquem o conhecimento que estão aprendendo na solução de problemas, sob a supervisão de um professor. Esse modelo é preponderante no início do curso, 
denominado "ciclo básico", e o mesmo se repete no ciclo pré-clínico, no qual o estudante continua recebendo conteúdo que nem sempre está contextualizado nas questões relevantes para a atenção e o cuidado em saúde.

A sala de aula invertida apresenta-se como uma alternativa para professores que desejam diversificar a sua forma de ensinar. Tem como diferencial o fato de favorecer a interação entre os próprios estudantes e destes com o professor, valorizando o momento do encontro na sala de aula para a discussão e a solução de questões e problemas relevantes para o aprendizado(3).

\section{SALA DE AULA INVERTIDA: BREVE HISTÓRICO}

O conceito da sala de aula invertida foi proposto inicialmente por Baker, na $11^{\text {a }}$ Conferência sobre Aprendizagem no Ensino Superior, que ocorreu na Flórida, no ano de $2000^{(4)}$. O trabalho de Baker propunha essencialmente a utilização de ferramentas de gerenciamento da aprendizagem pela internet. Lage et al. ${ }^{(5)}$, no mesmo período, chamaram a atenção para a importância do desenvolvimento de novas tecnologias e recursos multimídia que fossem acessíveis aos estudantes, em um ambiente favorável à integração deles ao processo de ensino e aprendizagem. Os autores destacam que, quando adequadamente utilizadas, as novas tecnologias motivam e entusiasmam os estudantes, nativos da "era digital", para o uso da internet, computadores, tablets e smartphones $^{(5)}$. Na proposta da sala de aula invertida, o uso de recursos multimídia é central e está associado ao emprego de outras estratégias de ensino que podem atrair e alcançar estudantes com diferentes estilos de aprendizagem ${ }^{(6)}$.

Outro autor que sempre é lembrado e cujo trabalho influenciou no desenho proposto para a sala de aula invertida é Eric Mazur, professor de Física Aplicada na Universidade de Harvard (EUA). Em 1991, Mazur revisou suas práticas de ensino, buscando tornar o ensino da física mais acessível aos estudantes. Para isso, decidiu envolvê-los diretamente em seu próprio aprendizado. Ao invés de manter as aulas teóricas habituais, ele elaborou uma proposta na qual os estudantes preparavam o tema da aula previamente (lendo capítulos do livro-texto do curso) e usavam o tempo da aula para trabalharem em grupos na solução de tarefas específicas elaboradas pelo professor. Essa proposta favorecia tanto a interação entre os estudantes quanto a aprendizagem colaborativa entre os pares (peer-learning). Por ela, o momento presencial sempre é dedicado ao trabalho em equipe e à discussão e à solução dos problemas propostos ${ }^{(7)}$.

Em 2006, Jonathan Bergmann e Aaron Sams, professores de química no Colorado, elaboraram uma proposta em que as aulas eram previamente gravadas e disponibilizadas aos estudantes, como tarefa prévia ao encontro que teriam na sala de aula. Durante a atividade presencial, discutia-se a solução de problemas em que os conceitos aprendidos eram necessários, sob a supervisão e com o apoio direto do professor ${ }^{(8)}$.

Todos os tópicos a seguir serão discutidos considerando a utilização da sala de aula invertida no contexto dos cursos de graduação da área da saúde.

\section{SALA DE AULA INVERTIDA: O QUE É?}

A sala de aula invertida é um modelo pedagógico no qual os elementos típicos de uma aula convencional (a apresentação do conteúdo pelo professor) são trabalhados à distância, antes do momento presencial. Quando os estudantes e o professor se encontram para a aula, esse tempo é empregado na discussão de casos clínicos, resolução de problemas, proposição de projetos que sejam relevantes, que auxiliem na compreensão do conteúdo e que deixem clara a sua relevância no contexto profissional.

O formato mais utilizado de material para estudo prévio é o da videoaula, que habitualmente é gravada pelo próprio professor. O professor pode também utilizar-se de materiais que já estão disponíveis na internet, desde que sejam de boa qualidade e se adequem ao próposito do curso e aos objetivos de aprendizagem. Até recentemente, a produção e a divulgação de videoaulas eram trabalhosas e restritas, entretanto, isto já é passado. Hoje existe grande facilidade para a produção de 
material educacional pelos professores e este pode ser consultado pelos estudantes de diferentes formas através de dispositivos móveis, em qualquer lugar e a qualquer hora. Moffett (2015), em um artigo sobre o tema, sugere diversos recursos que podem ser utilizados pelos docentes para disponibilizar conteúdo ou oportunidades de aprendizagem aos seus estudantes ${ }^{(9)}$. Alguns desses recursos e suas fontes estão resumidos na Tabela 1:

Tabela 1: Recursos disponíveis para serem utilizados na sala de aula invertida

\begin{tabular}{lll}
\hline \multicolumn{1}{c}{ Finalidade: } & \multicolumn{2}{c}{ Recurso/software } \\
\hline Produção de vídeos & $\checkmark$ & Camtasia Studio \\
Programas de apresentação & $\checkmark$ & Adobe Captivate \\
(pode-se gravar voz sobre os slides) & $\checkmark$ & Google Presenter \\
Podcasts & $\checkmark$ & Keynote \\
& $\checkmark$ & Microfone no telefone celular \\
Vídeos disponíveis on-line & $\checkmark$ & Audacity \\
& $\checkmark$ & ITunesU \\
Interface para compartilhar material didático com & $\checkmark$ & YouTube \\
O estudante & $\checkmark$ & Vimeo \\
& $\checkmark$ & Dropbox \\
& $\checkmark$ & Google Drive \\
Ambientes virtuais de aprendizagem & $\checkmark$ & Microsoft OneDrive \\
& $\checkmark$ & Moodle \\
& $\checkmark$ & Blackboard \\
Programas de comunicação síncrona & $\checkmark$ & Edmodo \\
\hline
\end{tabular}

A proposta da sala de aula invertida entrelaça diversos conceitos: o da aprendizagem ativa e colaborativa, da autonomia do estudante, da aprendizagem significativa e do estudo/educação à distância. Metodologias de ensino que associam estratégias de ensino presencial com momentos de estudo à distância costumam ser denominadas de aprendizagem híbrida (do inglês blended-learning). Nesse contexto, o professor atua muito mais como aquele que planeja e implementa a atividade de ensino (deixando claros quais os objetivos de aprendizagem a serem alcançados) e atua nos encontros presenciais como um facilitador da aprendizagem, garantindo através de estratégias de avaliação formativa e somativa que os resultados esperados foram realmente alcançados.

\section{SALA DE AULA INVERTIDA: COMO FUNCIONA?}

Não existe uma única forma de organizar uma atividade utilizando a estratégia da sala de aula invertida. De modo geral, o termo é empregado sempre que se disponibiliza uma aula previamente gravada, seguida por momentos presenciais de discussão e trabalho em grupo. 
Um dos grandes desafios para esse tipo de atividade é garantir que o estudante cumpra a tarefa pré-classe antes de vir para o encontro presencial. Existem algumas formas de estímulo para que esse objetivo seja alcançado. Uma das maneiras de garantia de preparo dos estudantes é elaborar um teste sobre a aula gravada para ser respondido antes da aula, com questões (geralmente de múltipla escolha) que podem ser respondidas em uma plataforma virtual de ensino, ou em papel como primeira atividade do momento presencial. A discussão e a devolutiva (feedback) do pré-teste são mais uma oportunidade de aprendizagem e devem ser consideradas pelo professor.

Durante o momento presencial, o professor deve atuar como facilitador da aprendizagem e estimular o trabalho dos estudantes na discussão e na solução de tarefas, problemas ou casos clínicos que são muito úteis e costumam atrair o interesse dos estudantes dos cursos da área da saúde. Esse tipo de exercício (resolver um caso clínico) requer do aluno mais que memorização - exige compreensão, análise e síntese do conteúdo que está sendo aprendido. Em um mesmo momento presencial, o professor pode formular diferentes atividades (estações) para que os estudantes façam um rodízio entre elas ${ }^{(10)}$.

\section{SALA DE AULA INVERTIDA: POR QUE UTILIZAR NA GRADUAÇÃO?}

Existe uma crença "equivocada" na educação das profissões da saúde de que só é possível mudar a forma de ensinar do professor mudando o currículo do curso. Muitos gestores acadêmicos, e infelizmente também vários professores, depositam na mudança curricular do curso a responsabilidade pela indução e pela revisão das práticas de ensino. Cada professor, a qualquer momento, pode e deve rever suas práticas de ensino, buscando torná-las mais adequadas aos propósitos do curso e efetivas para gerar competência entre os estudantes.

Além de contar com a iniciativa do próprio professor, caberá à instituição criar e oferecer oportunidades de capacitação e de desenvolvimento docente, como já está definido e recomendado pelas Diretrizes Curriculares dos Cursos de Graduação de Medicina, publicadas em 2014(11).

\section{SALA DE AULA INVERTIDA: QUAIS AS LIMITAÇÕES E OS DESAFIOS?}

Diferentemente da aula teórica centrada no professor, essa proposta requer mais tempo para o planejamento e cuidados na execução, especialmente no que diz respeito à elaboração do material de estudo prévio e das tarefas a serem executadas no momento presencial.

O erro mais comum na elaboração das videoaulas é a produção de material com qualidade técnica ruim e gravações muito longas (tempo maior do que 15 a 20 minutos), o que pode reduzir significativamente o interesse do estudante na fase preparatória.

A realização de um teste de conhecimento relacionado ao tema apresentado na videoaula é uma estratégia interessante para checar a compreensão (diagnóstico) e também oferecer esclarecimentos para aspectos que ainda não ficaram totalmente claros aos estudantes.

O professor também precisa ter uma noção básica sobre técnicas de facilitação do trabalho em grupo, que será o ponto alto durante os momentos presenciais. Apesar de não se tratar de nenhuma habilidade excepcional, é necessário lembrar que: nesta posição, o professor deve favorecer a interação entre os estudantes; antes de responder às questões, deve checar com os outros estudantes se eles têm alguma contribuição a dar; deve acompanhar as atividades de cada grupo, esclarecendo e ajudando os estudantes na solução da tarefa proposta e oferecendo feedback sempre que for necessário.

É essencial também que todos os estudantes tenham acesso aos recursos mínimos de informática para que possam cumprir com as demandas de preparação pré-classe (assistir às videoaulas, por exemplo). 


\section{SALA DE AULA INVERTIDA: COMO ENGAJAR OS ESTUDANTES NO ESTUDO PRÉ-CLASSE?}

Solicitar ao estudante que prepare algum material ou realize tarefas preparatórias antes do momento presencial na sala de aula é tão antigo quanto desafiante, porém, nem sempre, o objetivo do professor é alcançado. Este é um desafio particularmente importante que o professor deve cuidar e superar junto com seus estudantes. Algumas estratégias já têm sido descritas neste sentido, por exemplo, na aprendizagem baseada em equipes (team-based learning-TBL), que propõe que seja feita uma avaliação individual e em equipes como parte das ações que buscam obter a "garantia de preparo" por parte do estudante. Nessa proposta de aprendizagem baseada em equipes, o professor realiza testes de múltipla escolha sobre o assunto que será discutido na atividade do dia, e o resultado compõe os itens a serem considerados na avaliação do próprio estudante ${ }^{(12)}$.

O uso de metodologias ativas de aprendizagem, entre elas a sala de aula invertida, implica uma mudança de postura do estudante, que deve ser o protagonista da sua própria aprendizagem. Dentre as dificuldades que o aluno enfrenta para realizar tarefas preparatórias, está a falta de tempo, a forte cultura de que a única forma de aprendizagem são as aulas teóricas ministradas presencialmente pelo professor e a necessidade de o estudante sair de sua zona de conforto ${ }^{(13)}$.

Entretanto, uma vez que o professor decida experimentar a proposta da sala de aula invertida, é necessário que ele pense também como poderá estimular e ter sucesso com seus estudantes na fase preparatória para a aula presencial. Os aspectos a serem considerados para o engajamento dos estudantes é que as tarefas sejam disponibilizadas, com antecedência, para favorecer o estudo no próprio ritmo do aluno, que terá acesso fácil e irrestrito ao material para que possa consultá-lo tantas vezes quanto forem necessárias ${ }^{(14)}$.

Bergmann \& Sams, em seu livro Sala de aula invertida, propõem a criação de vídeos para os alunos. Os vídeos podem ser domésticos, usando softwares de captura de tela. Os autores sugerem que os vídeos devem ser curtos, que os professores falem com entusiasmo, que sejam claros em relação ao assunto que será estudado e que os docentes acrescentem anotações e chamadas, para tornar o vídeo mais atrativo(8).

Existe uma preocupação em relação ao uso exclusivo de videoaula levar a um menor engajamento dos estudantes nas atividades pré-classe. Alunos mais "visuais e auditivos" devem se adaptar melhor à proposta de videoaulas, enquanto alunos mais cinestésicos podem preferir outros tipos de atividades, como atividades práticas ${ }^{(15)}$.

Sherma e cols (2015), ao utilizarem a estratégia da sala de aula invertida, incluíram outros recursos além do vídeo, tais como textos impressos e áudios (podcasts). Esses autores também ressaltam que não existe a necessidade de uma grande produção do material que será utilizado à distância, mas sim que esse material seja elaborado com o intuito principal de engajar os estudantes, sem provocar uma sobrecarga de informações. Os autores ressaltam também que o material produzido para a fase preparatória deve ser capaz de instrumentalizar o estudante para a discussão e a solução de problemas (casos-clínicos) durante o momento presencial(14),(16).

A produção de videoaulas tem sido a estratégia mais comumente preconizada pela literatura no tocante ao momento pré-classe ${ }^{(14)}$. Bishop \& Verlenger (2013) demonstram que o uso de videoaulas é superior ao da leitura dos livros-texto na sala de aula invertidaa ${ }^{(17)}$. Simon Li (2015) propõe, além do uso de vídeos, a integração dos alunos em um ambiente virtual, como o Moodle ou o EdModo(18).

Moffett (2015), sugere que os docentes não familiarizados com a produção de videoaulas podem utilizar vídeos já existentes na internet (YouTube ou Vimeo) ou podem gravar a voz sobre uma apresentação de slides. Outra estratégia, além das citadas, é o uso de blogs para fornecer material aos alunos, associados a um espaço em que eles possam colocar suas anotações ${ }^{(9)}$.

O envolvimento de estudantes de anos seguintes como monitores (peer-instruction) pode ser também uma estratégia para tornar o momento on-line mais colaborativo(16). 
$\mathrm{Na}$ Tabela 2, seguem os pontos centrais da estratégia da sala de aula invertida e questionamentos (adaptado de Sams et al.) que o professor deve fazer para checar a adequação da técnica que está utilizando(19):

Tabela 2: Aspectos centrais da estratégia da sala de aula invertida e questionamentos para checar a adequação da técnica*.

\section{Os quatro pilares da Sala de Aula Invertida}

Flexible: ser flexível e favorecer o uso de diferentes modos de ensino/aprendizagem;

organizar o espaço físico, de modo a facilitar o

F trabalho em grupo; o professor deve compreender que estudantes aprendem em tempos diferentes, e assim acomodar essas diferenças na medida do possível, durante as atividades realizadas no momento presencial.

Learning: nutrir a cultura de aprendizagem. 0 professor não deve ser mais a fonte primária e única da aprendizagem. O aprendizado deve estar centrado

L no estudante e na sua relação com os pares (peerlearning). O trabalho em sala de aula deve favorecer atividades que aprofundem e deem significado ao que está sendo aprendido.

Intentional teaching actions: o professor deve ter intencionalidade naquilo que faz e propõe aos estudantes. As metas devem estar sempre relacionadas aos objetivos de aprendizagem

I esperados, ao longo e após a conclusão do curso/ disciplina; o professor deve determinar o que deverá ser ensinado/aprendido e quais os recursos necessários para que isto aconteça; planejamento é fundamental, assim como contextualizar o que está sendo aprendido!

Professional Educator: os professores devem avaliar e prover feedback regular aos estudantes; devem refletir sobre a sua prática; devem aceitar uma certa "desordem controlada" na sala de aula, enquanto

P os estudantes aprendem uns com os outros nas discussões e resolução das tarefas/problemas propostos; apesar de ter menos visibilidade na sala de aula invertida, o professor continua tendo um papel central no processo de ensino e aprendizagem. $\checkmark \quad$ Estabeleço uma agenda que favoreça interação e reflexão por parte dos estudantes?

$\checkmark \quad$ Observo e acompanho continuamente estudantes para ajudá-los a fazerem ajustes, na medida do necessário?

$\checkmark \quad$ Ofereço aos estudantes diferentes
oportunidades de aprender o conteúdo?

$\checkmark \quad$ Ofereço ao estudante oportunidades de estar no centro do próprio aprendizado, engajando-o em atividades relevantes e significativas para a sua aprendizagem?

$\checkmark \quad$ Deixo essas atividades continuamente acessíveis a todos os estudantes, sempre oferecendo feedback?

$\checkmark \quad$ Priorizo conceitos que foram apresentados na aula gravada para serem discutidos no presencial pelos estudantes?

Crio e atualizo videoaulas relevantes e de boa qualidade para os estudantes?

$\checkmark \quad$ Preparo material didático diferenciado e capaz de alcançar todos os estudantes?

Fico disponível para os estudantes (individual, em grupos) na medida em que é necessário?

$\checkmark \quad$ Faço avaliação formativa e dou feedback regular aos estudantes para informá-los sobre como poderão melhorar no futuro?

$\checkmark$ Colaboro e reflito com outros educadores, assumindo a responsabilidade por revisar e melhorar minha prática docente?

*Adaptado de Sams et al. (2014) (19) 


\section{SALA DE AULA INVERTIDA: USANDO A ESTRATÉGIA NA ÁREA DA SAÚDE}

A sala de aula invertida pode ser empregada nas diferentes situações no ensino da saúde, seja nos anos iniciais, seja nos profissionalizantes.

Um estudo comparativo foi realizado com estudantes de anatomia da Universidade de Utah. Parte dos estudantes permaneceu aprendendo anatomia através de aulas tradicionais, enquanto um segundo grupo participou de um curso que utilizou a sala de aula invertida. O período de intervenção foi de 17 semanas. Ao final, uma avaliação, baseada nos domínios cognitivos da Taxonomia de Bloom, foi realizada. Os estudantes que participaram do grupo da sala de aula invertida apresentavam níveis de conhecimento semelhantes aos da aula tradicional, porém a capacidade de análise desses estudantes era significantemente maior quando esta era avaliada, demonstrando que eles eram mais competentes para resolver problemas (níveis cognitivos mais altos na Taxonomia de Bloom) ${ }^{(20)}$. Em estudo semelhante, Fermozelli (2016) fez uma sobreposição da sala de aula invertida e do aprendizado baseado em problemas com alunos do $2^{\circ}$ ano de Medicina. Nesse estudo, houve aumento no número de conceitos satisfatórios em relação aos anos anteriores. A análise do discurso coletivo dos alunos mostrou que eles tiveram uma percepção positiva da intervenção(21).

Em uma coorte de estudantes de saúde pública, a introdução do da sala de aula invertida não produziu melhora nos escores em uma avaliação quando comparados a alunos que aprenderam através de aulas expositivas. Porém, os escores obtidos foram maiores que nos anos anteriores. Esses alunos também relataram, em grupos focais, que tiveram uma percepção positiva do aprendizado, com maior interação com os pares e com os seus instrutores ${ }^{(22)}$.

Aproximadamente 100 estudantes de Farmácia aprenderam conceitos de arritmias cardíacas (ciências básicas, farmacologia e terapêutica) em sala de aula invertida e foram comparados com um grupo de alunos que estudaram esses mesmos conceitos através de aulas tradicionais. O desempenho dos alunos que aprenderam arritmias por sala de aula invertida foi $30 \%$ maior em farmacologia e $15 \%$ maior em terapêutica ${ }^{(23)}$.

Pierce e Fox utilizaram a sala de aula invertida em uma disciplina de farmacoterapia renal. Como resultado dessa intervenção, obtiveram melhor desempenho dos alunos nas avaliações. No questionário de satisfação, verificaram percepções favoráveis sobre a intervenção utilizada. Os autores relacionam os vários fatores que podem ter contribuído para o resultado positivo na intervenção, dentre eles: o contato com o material do curso antes da aula, as avaliações formativas feitas durante o módulo e as atividades interativas sob a supervisão do professor na classe(24).

Em alunos do curso de Enfermagem, Critz e Knight verificaram que a troca de um modelo de aula tradicional para um modelo de sala de aula invertida fazia com que os alunos estudassem mais tempo porque se sentiam mais motivados. Também observaram uma melhora no aproveitamento dos alunos nas avaliações ${ }^{(25)}$.

Em um curso de Odontologia, na comparação de duas metodologias ativas, o TBL e a sala de aula invertida produziram resultados semelhantes no aprendizado sobre prótese dentária. Além disto, as duas estratégias foram superiores ao grupo que aprendeu essa disciplina de maneira tradicional(26).

No curso de Medicina da Universidade de Michigan, a adoção da sala de aula invertida em uma disciplina de ginecologia e obstetrícia mostrou que os alunos que participaram da intervenção ficaram mais satisfeitos. O desempenho nas questões do teste da National Board of Medical Examiners foi semelhante(27). Em outras situações, no curso de Medicina, o uso da sala de aula invertida também promoveu um maior engajamento dos alunos e potencializou o aprendizado em humanidades(28), cirurgia $^{(29)}$ e medicina de urgência(30).

\section{CONCLUSÕES}

A sala de aula invertida é uma metodologia ativa de ensino e aprendizagem, pois confere ao estudante o protagonismo de seu aprendizado. 
Também fornece ao estudante a possibilidade de aprender no seu próximo ritmo, bem como o acesso ao material tantas vezes quanto julgar necessário.

O uso de tecnologia e da internet favorece o engajamento do estudante.

A aplicação do conhecimento adquirido, na presença do professor, favorece a detecção de lacunas e fragilidades do estudante e oportuniza o pronto feedback.

A capacitação docente e o apoio institucional são fundamentais para a implantação dessa estratégia pedagógica nos cursos da saúde.

\section{REFERÊNCIAS}

1. MCLEAN, M; CILLIERS, F; VAN WYK, JM. Faculty development: Yesterday, today and tomorrow. Med. Teach., janeiro de 2008;30(6):555-84.

2. GORMALlY, C; EVANS, M; BRICKMAN, P. Feedback about Teaching in Higher Ed: Neglected Opportunities to Promote Change. Cell Biol. Educ., 1 de junho de 2014;13(2):187-99.

3. O'FLAHERTY, J; PHILLIPS, C. The use of flipped classrooms in higher education: A scoping review. Internet High Educ., abril de 2015;25:85-95.

4. BAKER, J. W. (2000, April). The "classroom flip": Using web course management tools to become the guide by the side. In J. A. Chambers (Ed.), Selected papers from the 11th International Conference on College Teaching and Learning (11th, Jacksonville, Florida, April 12-15, 2000) (pp. 9-17). Jacksonville, FL: Center for the Advancement of Teaching and Learning.

5. LAGE, MJ; PLATT, GJ; TREGLIA, M. Inverting the Classroom: A Gateway to Creating an Inclusive Learning Environment. J. Econ. Educ. 2000;31(1):30.

6. COFFIELD, F. Learning and Skills Research Centre (Great Britain). Learning styles and pedagogy in post-16 learning: a systematic and critical review. London: Learning and Skills Research Centre; 2004 [citado 15 de agosto de 2016]. Available at: https://www.Isneducation.org.uk/user/NoCookies.aspx?ReturnUrl=/user/order.aspx?code=041543\&src=xoweb\&cookie_ test=true

7. MAZUR, E. Peer instruction: a user's manual. Upper Saddle River, N.J: Prentice Hall; 1997. 253 p. (Prentice Hall series in educational innovation).

8. BERGMANN, J; SAMS, A. Flip your classroom: reach every student in every class every day. Eugene, Or: International Society for Technology in Education; 2012. 112 p.

9. MOFFETT, J. Twelve tips for "flipping" the classroom. Med Teach. 3 de abril de 2015;37(4):331-6.

10. WAKABAYASHI, N. [Flipped classroom as a strategy to enhance active learning]. Kökübyö Gakkai Zasshi J Stomatol Soc Jpn. março de 2015;81(3)-82(1):1-7.

11. BRASIL, Ministério da Educação. Diretrizes Curriculares Nacionais (DCNs) do Curso de Graduação em Medicina. 2014. Available at: http://portal.mec.gov.br/index.php?option=com_docman\&view=download\&alias=15514-pces116-14\&ltemid=30192

12. BOLLELA, VR; SENGER, MH; TOURINHO, FSV; AMARAL, E. Aprendizagem baseada em equipes: da teoria à prática. Med. Ribeirão Preto On-line, 3 de novembro de 2014;47(3):293.

13. EDGINTON, A; HOLBROOK, J. A Blended Learning Approach to Teaching Basic Pharmacokinetics and the Significance of Face-to-Face Interaction. Am. J. Pharm. Educ., setembro de 2010;74(5):88. 
14. SHARMA, N; LAU, CS; DOHERTY, I; HARBUTT, D. How we flipped the medical classroom. Med. Teach., 3 de abril de 2015;37(4):327-30.

15. WILLIAMS, DE. The Future of Medical Education: Flipping the Classroom and Education Technology. Ochsner J. 2016;16(1):14-5.

16. ESTES, M; INGRAM, R; LIU, J. A Review of Flipped Classroom Research, Practice, and Technologies. Int. High Educ. Teach Learn. 2014;4. Available at: https://www.hetl.org/a-review-of-flipped-classroom-research-practice-and-technologies/

17. BISHOP, J; VERLEGER, MA. The Flipped Classroom: A Survey of the Research Paper. Present 2013 ASEE Annu Conf. Atlanta Ga. 2013; Available at: https://peer.asee.org/22585

18. LI, S. Flip the classroom. Educ. Prim. Care, 2 de novembro de 2015;26(6):438-40.

19. SAMS, A; BERGMANN, J; DANIELS, K; BENNET, B; MARSHALL, H; ARFSTROM, K. What Is Flipped Learning?. 2014. Available at: http://flippedlearning.org/wp-content/uploads/2016/07/FLIP_handout_FNL_Web.pdf

20. MORTON, DA; COLBERT-GETZ, JM. Measuring the impact of the flipped anatomy classroom: The importance of categorizing an assessment by Bloom's taxonomy: Impact of the Flipped Anatomy Classroom. Anat. Sci. Educ., julho de 2016 [citado 15 de agosto de 2016]. Available at: http://doi.wiley.com/10.1002/ase.1635

21. FERMOZELLI JA. Estratégias de Blended-Learning no ensino de patologia geral em um curso de medicina [Mestrado]. 2016. Sorocaba, São Paulo: Pontifícia Universidade Católica de São Paulo.

22. GALWAY, LP; CORBETT, KK; TAKARO, TK; TAIRYAN, K; FRANK, E. A novel integration of online and flipped classroom instructional models in public health higher education. BMC Med. Educ., dezembro de 2014 [citado 15 de agosto de 2016];14(1). Available at: http://bmcmededuc.biomedcentral.com/articles/10.1186/1472-6920-14-181

23. WONG, TH; IP, EJ; LOPES, I; RAJAGOPALAN, V. Pharmacy Students' Performance and Perceptions in a Flipped Teaching Pilot on Cardiac Arrhythmias. Am. J. Pharm. Educ., dezembro de 2014;78(10):185.

24. PIERCE, R; FOX, J. Vodcasts and Active-Learning Exercises in a "Flipped Classroom" Model of a Renal Pharmacotherapy Module. Am. J. Pharm. Educ., dezembro de 2012;76(10):196.

25. CRITZ, CM; KNIGHT, D. Using the Flipped Classroom in Graduate Nursing Education: Nurse Educ. 2013;38(5):210-3.

26. NISHIGAWA, K; OMOTO, K; HAYAMA, R; OKURA, K; TAJIMA, T; SUZUKI, Y, et al. Comparison between flipped classroom and team-based learning in fixed prosthodontic education. J. Prosthodont. Res., maio de 2016 [citado 15 de agosto de 2016]; Available at: http://linkinghub.elsevier.com/retrieve/pii/S1883195816300275

27. MORGAN, H; MCLEAN, K; CHAPMAN, C; FITZGERALD, J; YOUSUF, A; HAMMOUD, M. The flipped classroom for medical students. Clin. Teach., junho de 2015;12(3):155-60.

28. GROSSMAN, E; GROSSEMAN, S; AZEVEDO, GD; FIGUEIRÓ-FILHO, EA; MCKINLEY, D. Flipped classroom on humanities: medicine, narrative and art. Med. Educ., novembro de 2015;49(11):1142-1142.

29. LIEBERT, CA; MAZER, L; BEREKNYEI MERRELL, S; LIN, DT; LAU, JN. Student perceptions of a simulation-based flipped classroom for the surgery clerkship: A mixed-methods study. Surgery, setembro de 2016;160(3):591-8.

30. ROSE, E; CLAUDIUS, I; TABATABAI, R; KEARL, L; BEHAR, S; JHUN, P. The Flipped Classroom in Emergency Medicine Using Online Videos with Interpolated Questions. J. Emerg. Med., julho de 2016 [citado 15 de agosto de 2016]. Available at: http://linkinghub.elsevier.com/retrieve/pii/S0736467916301639 